\title{
Estudio de caso con utilización del instrumento de Katharine Kolcaba teoría de rango medio del confort
}

\author{
Guadalupe Nava Galán
}

RESUMEN

El estudio de caso; determina el grado de comodidad de la persona hospitalizada y cómo la familia aplica medidas necesarias para que está se lleve a cabo en el Servicio de Neurocirugía del Instituto Nacional de Neurología y Neurocirugía (INNN) para que el paciente con daño neurológico tenga confort, la muestra esta constituida por una persona con diagnóstico de meningioma grado I. Se utilizó instrumento validado de la teoría de mediano rango, cuestionario general de comodidad (GCQ), desarrollado por la doctora Katharine Kolcaba, se determinaron las necesidades después de la intervención de la familia, quiénes fueron orientado hacia las medidas que podían realizar sin poner en peligro la vida del PE. Altos grados de comodidad fueron encontrados, después de la intervención. Material y métodos: proceso enfermero. Estos hallazgos sugieren que la familia está en capacidad de asumir determinadas responsabilidad en el cuidado del paciente, ayudándolo a afrontar su crisis de enfermedad y favoreciendo de esta manera un cuidado más humano y holístico en los hospitales, siendo un apoyo indispensable para fortalecer el cuidado y mejor evolución del tratamiento.

Palabras clave: estudio de caso, confort, comodidad.

\section{Case study on the use of instrument of Katherine Kolcaba middle range theory: from comfort}

Case study. Establish the comfort level of the hospitalized person, and how the family applies the necessary gears to the comfort being made in the neurosurgery service, from the INNN, for the person with neurological damage has comfort, the sample is constituted by one person with Meningioma level 1. It was used a valid instrument: the medium range theory, named general comfort questionnaire (GCQ); developed by the MD. Katherine Kolcaba, it were determinate the necessities after the intervention of the family, whose was oriented in which applies could be realized without endangering life. High levels of comfort were founded, after the intervention. The methodology was the nursing process. These findings suggest that the family has the capacity of assume determinate responsibilities in the person care, helping him to face the disease crisis and favoring the humanitarian and holism care in the hospitals, being an indispensable support to encourage the care and a better treatment evolution.

Key word: case study, comfort, convenience.

Correspondencia: Ma. Guadalupe Nava Galán, Instituto Nacional de Neurología y Neurocirugía. Insurgentes Sur \# 3877. Col. La Fama.

14269 México, D.F. E-mail: mgngalan@hotmail.com.

\section{MARCO DE REFERENCIA}

Katharine Kolcaba nació en Clevelan, Ohio en 1944; en 1965 se diplomó en enfermería en St. Luke’s Hospital de Clevelan. Trabajo tiempo parcial durante algunos años como enfermera medicoquirúrgica, considerando el cuidado prolongado y cuidado domiciliario. En 1987, consiguió la primera licenciatura superior que se realizaba en enfermería en la escuela de Enfermería Frances Payne Bolton, de la Case Western Reserve University (CWRU). Estudio una especialidad en gerontología, mientras la llevaba a cabo, asumió el cargo de enfermera en jefe de la unidad de demencia. En dicha unidad, empezó a teorizar acerca de la comodidad o confort. Después de realizar una maestría en enfermería, trabajo como profesora en la University of Akron College of Nursing. Desde entonces, posee el certificado de gerontología de la American Nurses Association (ANA). Volvió a la CWRU para realizar un doctorado en enfermería a tiempo parcial mientras continuaba trabajando como profesora de tiempo completo. Durante los siguientes 10 años, utilizó el trabajo de su doctorado para 
desarrollar una teoría y demostrarla; publicó un análisis del concepto del confort con su esposo, el filósofo (Kolcaba \& Kolcaba, 1991) quien elaboró un diagrama con los aspectos de la comodidad (Kolcaba, 1991), utilizó la comodidad como objetivo del cuidado (Kolcaba, 1992ª), contextualizó la comodidad en una teoría de nivel intermedio (Kolcaba, 1994) y demostró la teoría en un estudio de intervención (Kolcaba \& Fox, 1999). En 1995 recibió un premio honorífico como investigadora de la Midwest Nursing Research Society del Lillian De Young Resarch Award del University of Ackron College of Nursing, por su labor sobresaliente en el desarrollo de la investigación, se doctoró en enfermería en 1997 y recibió el certificado de autoridad (especialista en enfermería clínica) al mismo tiempo. Así mismo recibió el Marie Haug Student Award por su excelente trabajo en estudios anteriores en la CWRU, es miembro de la ANA Society of Scholars y aparece en el Who's Who in American Nursing (1991) y en The Enciclopedia of Nursing Research (Kolcaba, 1998). En la actualidad, Kolcaba es profesora asociada de enfermería en la University of Akron College of Nursing, en donde enseña teoría e investigación en enfermera. Es fundadora y coordinadora del programa de enfermería parroquial local, y miembro de la ANA, la Sigma Theta Tau, la Midwest Nursing Research Society, y la Health Ministrie Association y League of Women Voters ${ }^{1}$.

\section{Fuentes teóricas}

Kolcaba inició su trabajo teórico elaborando un diagrama de la práctica enfermera para su doctorado. Cuando presento su marco para el cuidado de demencia (Kolcaba, 1992ª). El primer paso el análisis del concepto, empezó con una amplia revisión de la bibliografía existente sobre comodidad relacionada a la enfermería, medicina, psicología, psiquiatría y ergonomía. Shakespeare y el Oxford English Dictionary, relata el origen de esa palabra, ofreció una visión histórica de la comodidad en enfermería por Nightingale quién 1859 declaró: nunca debemos olvidarnos del objetivo de la observación. La observación no sirve para recopilar datos variados y hechos curiosos, sino para salvar vidas y promover salud y comodidad. De 1900 a 1929, la comodidad fue el centro de la enfermería y la medicina, y a través de ella se llegaba a la recuperación (Mcllveen \& Morse, 1995).

La enfermera está obligada a prestar atención a los detalles que influyen en la comodidad de la persona, a la hora de procurarla, no hay que ignorar ningún detalle, pues es el primer y último objetivo de la enfermera hacer que las personas se sientan cómodas, Aikens, 1908. Harmer 1926 afirmó que el cuidado enfermero consistía en proporcionar un ambiente general de comodidad, el cuidado personal incluye prestar atención a la felicidad, comodidad, tranquilidad tanto física como mental, además del descanso y sueño, nutrición, la higiene y la eliminación «. Goodnow (1935) dedicó un capítulo de su libro The Technique of Nursing a la comodidad de la persona. Escribió siempre se juzga a una enfermera por su capacidad para hacer que el paciente se sienta cómodo. La comodidad es física y mental, y la responsabilidad de la enfermera va más allá del cuidado físico. En libros de texto de 1904, 1914 y 1919, se describe que la comodidad emocional se denominó comodidad mental y dependía principalmente de la comodidad física y de la modificación del entorno de los pacientes (Mcllveen \& Morse, 1995).

Con estos ejemplos, la comodidad, es algo positivo. Se consigue con la ayuda de las enfermeras y, en algunos casos, indica una enfermedad o un estado previo. Intuitivamente, se asocia con la actividad de cuidado.

Desde el principio, Kolcaba explicó sus características principales y a partir de la ergonomía, la relación directa que se establece entre comodidad y relación de una tarea. Sin embargo, su significado suele estar implícito, oculto en el contexto, y suele resultar ambiguo. El concepto varía semánticamente según sea verbo, nombre, adjetivo, adverbio, proceso o resultado.

Para sintetizar y elaborar los tipos de confort del análisis conceptual de Kolcaba, se utilizaron las primeras obras de tres de las primeras teórias enfermeras (Kolcaba \& Kolcaba, 1991). El alivio se elaboró a partir del trabajo de Orlando (1961) quien afirmaba que las enfermeras satisfacían las necesidades de los pacientes. Para la tranquilidad, se utilizó el trabajo de Henderson (1966), que describía las trece funciones básicas de los seres humanos que debían mantenerse en la homeóstasis. La trascendencia se redacto a partir de la obra de Paterson y Zderad (1975), quienes creían que podían superar las dificultades con la ayuda de las enfermeras. En su teoría del cuidado, Watson (1979) declaró que el entorno de los pacientes era muy importante para su bienestar mental y físico. Por tanto, siempre que fuera posible, las enfermeras debían proporcionar comodidad a través de intervenciones ambientales. Watson identificó las medidas de comodidad que las enfermeras utilizaban al respecto (Who's Who in American Nursing, 1991); y utilizó el término medidas de confort como sinónimo de intervenciones.

\section{Problemática}

Las personas cuando presentan alguna clase de alteración en su salud se ven en la necesidad de permanecer hospitalizadas, originando tanto en ellas mismas como en su familia una gran incomodidad interrumpiendo su función familiar. La persona asume un papel pasivo. En muchas ocasiones, la familia queda aislada y desinformada acerca de su enfermo y no se toma en cuenta en el cuidado del mismo. 


\section{Preguntar e investigar}

¿Cuál es la comodidad de la persona hospitalizada en el Servicio de Neurocirugía, y cuando se preveen medidas de comodidad que involucran a la familia?

\section{Propósito}

Orientar a la familia a proporcionar comodidad a la persona hospitalizada para favorecer la tranquilidad y el bienestar a través de intervenciones de enfermería, que están encaminadas a aumentar el confort de la persona, dando un giro total al enfoque del cuidado biológico y pasando a un enfoque centrado en la persona y su familia; así como, a hospitales más humanos y cálidos, donde la familia y el enfermo se sientan como en su hogar y la situación de enfermedad no sea tan notoria ni estresante. Esto se puede lograr involucrando a la familia en el cuidado para que sea un miembro activo y no sólo un visitante pasivo.

\section{OBJETIVOS}

- Proporcionar comodidad a la persona hospitalizada.

- Educar al familiar para procurar confort.

\section{Conceptos principales y definiciones}

En la teoría del confort de Kolcaba, los receptores de las medidas de confort tienen diferentes orígenes tales como: pacientes, estudiantes, prisioneros, trabajadores, ancianos, comunidades e instituciones ${ }^{2}$.

\section{Necesidad de cuidados en la salud}

Kolcaba define las necesidades de cuidados a la salud como necesidades para conseguir la comodidad, a partir de situaciones estresantes de cuidado para la salud que los sistemas tradicionales de apoyo de los receptores no pueden satisfacer. Incluyen necesidades físicas, psicoespirítuales, sociales y ambientales, que se observan a través del seguimiento y de los informes verbales y no verbales, así como necesidades relacionadas con los parámetros fisiopatológicos, educación, apoyo, asesoramiento e intervención financiera .

\section{Medidas de confort}

Pueden definirse como intervenciones enfermeras las diseñadas para satisfacer necesidades específicas de comodidad de las personas receptoras de cuidados por ejemplo, necesidades fisiológicas, sociales, económicas, psicológicas, espirituales, ambientales y físicas ${ }^{3}$.

\section{Variables de intervención}

Las variables de intervención pueden definirse como fuerzas de interacción que influyen en la percepción por parte de los receptores de la comodidad total. Estas variables pueden ser las experiencias pasadas, edad, actitud, estado emocional, sistema de apoyo, el pronóstico, economía y la totalidad de elementos de la experiencia de los receptores.

\section{Confort}

El confort o comodidad se define como el estado que experimentan los receptores de las medidas para proporcionarles comodidad. Consistente en la experiencia inmediata y holística de fortalecerse gracias a la satisfacción de las necesidades de los tres tipos de comodidad son: alivio, tranquilidad y trascendencia ${ }^{4}$ que se definen cómo:

- Alivio: el estado de un receptor que ha visto cumplida una necesidad específica, se refiere a la satisfacción de necesidades; es decir, se deben aliviar malestares en los pacientes.

- Tranquilidad: el estado de calma o satisfacción, hace referencia a la persona entera, a la comodidad en la mente y cuerpo, estado de satisfacción y positivo.

- Trascendencia: estado en el cual un individuo está por encima de sus problemas o dolor. (Kolcaba 2003), es independiente y se refiere a las medidas de comodidad que fortalecen y engrandecen a una persona. La persona surge por encima del dolor o problemas se describió con los contextos sobre holismo, como:

Los cuatro contextos de la experiencia (físico, psicoespiritual, social y ambiental) (Kolcaba, 1994; Kolcaba \& Fox, 1999). Los tipos de comodidad pueden definirse como (Kolcaba, 2001):

1. Físico: perteneciente a las sensaciones corporales.

2. Psicoespirítual: perteneciente a la consciencia interna del yo, como: autoestima, autoconcepto, sexualidad, y el significado de la vida, en relación con un orden o estado superior.

3. Social: se refiere a las relaciones interpersonales, familiares y sociales.

4. Ambiental: perteneciente al entorno y condiciones e influencias externas. como: luz, ruido, temperatura, entre otros, y como se interrelacionan los sentidos con los contextos surgiendo una estructura taxonómica, en la cual la comodidad de los pacientes es experimentada en un 
contexto y sentido específico. Dicha estructura taxonómica permitió a Kolcaba el desarrollo del Cuestionario General de Comodidad (GCQ).

Conductas de búsqueda de la salud.

La doctora Rozella Schlotfeldt (1975); describió el concepto de las conductas de búsqueda de la salud como aquellas que representan las acciones relacionadas con la consecución de la salud, como la define el receptor o receptores, después de consultar con la enfermera estas conductas pueden ser internas, externas o enfocadas a conseguir una muerte tranquila.

\section{Integridad institucional}

Son corporaciones, comunidades, escuelas, hospitales, iglesias y reformatorios; entre otros, que poseen propiedades que hacen sentirse completo, total, razonable, recto, atrayente, honesto y sincero. Esta definición se elaboró a partir de la bibliografía de la investigación de los resultados, e incluye elementos normativos y descriptivos. La institución puede poseer muros, pero no los necesita.

\section{Utilización de pruebas empíricas}

La investigación moderna sobre la comodidad empezó en la década de 1980, cuando se inicio un período de reconocimiento colectivo; aunque con estudios individuales del concepto de la comodidad holística. Morse (1983) empezó a observar las acciones de confort de las enfermeras, y describió el término como la acción enfermera más importante para proporcionar cuidado enfermero a los enfermos. Hamilton (1989) dió un paso adelante estudiando lo que la comodidad representa desde la perspectiva del enfermo, utilizando entrevistas en un centro de cuidado prolongado para saber cómo cada paciente definía la comodidad 5 .

El cuidado cómodo surge de una situación de estímulos y tensiones negativas, que originan necesidades de comodidad en intervenciones para satisfacer dichas necesidades, moviendo la tensión en una dirección positiva. El paciente percibe si la tensión cambia por aplicación de intervenciones. La enfermera evalúa si la comodidad fue alcanzada por medio de valoración de la percepción del paciente, tanto objetiva como subjetivamente ${ }^{6}$. La enfermera determina si las medidas de comodidad implementadas aumentaron o sí se tiene que implementar nuevas. Estas medidas pueden ser muy sencillas como por ejemplo, mantener a la persona limpia, seca, en una buena posición, ofrecer alimentos, brindar un ambiente fresco, abrir ventanas o cerrarlas, manejar la luz, eliminar ruidos, proporcionar privacidad, permitir que tome sus propias decisiones, facilitar el acceso a la familia y enseñarles como proveer cariño físico y cuidado emocional; todo lo cual facilitará que la persona se fortalezca durante una situación de cuidado de la salud ${ }^{7}$.
Los resultados de las pruebas de cada principio se utilizaron por medio del estudio de Kolcaba y Fox (1999) en mujeres con cáncer de mama que se respaldaban mutuamente. Otras áreas de estudio que se han tratado con Kolcaba, incluyen unidades de quemados, hogares enfermeros, atención domiciliaria, dolor crónico, masaje terapéutico, pediatría, oncología, higiene dental, enfermería del transporte, cárceles y discapacidades mentales ${ }^{8}$.

\section{Conceptos metaparadigmáticos}

Enfermería: incluye valoración de las necesidades de la comodidad, diseño de las medidas para satisfacer esas necesidades y reevaloración de los niveles de después de la implantación de dichas medidas, en comparación con la situación anterior. La valoración y revaloración puede ser intuitiva y/o subjetivas, por ejemplo cuando una enfermera le pregunta aún paciente si está cómodo, u objetivas, cuando observa la curación de una herida o cambios en los valores analíticos o conducta vales. La valoración puede realizarse mediante la administración de escalas visuales analógicas o cuestionarios tradicionales, ambos sistemas desarrollados por Kolcaba (2001).

Paciente: los receptores del cuidado pueden ser individuos, familias, instituciones o comunidades que necesitan cuidados de salud.

Entorno: cualquier aspecto del paciente, familia o instituciones que la enfermera o familiares pueden manipular para que se sienta más cómodo.

Salud: es el funcionamiento óptimo de la persona, familiar o comunidad.

\section{Supuestos}

1. Los seres humanos ofrecen respuestas holísticas a estímulos complejos.

2. La comodidad es un objetivo holístico deseable que concierne a la disciplina enfermera.

3. Los seres humanos se esfuerzan por satisfacer sus necesidades básicas de comodidad o para que alguien las satisfaga, tratandose de un esfuerzo activo.

4. El aumento de la comodidad apremia a los pacientes a escoger conductas en búsqueda de la salud.

5. Las personas que están autorizados a participar activamente en estas conductas se muestran satisfechos con su cuidado de salud.

6. La integridad institucional se basa en un sistema de valores orientado a los receptores del cuidado ${ }^{9}$.

\section{Afirmaciones teóricas}

1. Las enfermeras identifican las necesidades de confort no satisfechas de sus enfermos, diseñando medidas para 
satisfacerlas potenciando su comodidad, lo que constituye el resultado inmediato deseado.

2. La potenciación de comodidad está directa y positivamente relacionada con implicación en las conductas de búsqueda de la salud, lo que constituye el siguiente resultado deseado.

3. Cuando las personas reciben ayuda adecuada para realizar las conductas saludables por sí solas, como rehabilitación, con y/o el programa de recuperación; así como régimen también se potencializa la integridad estructural (Kolcaba, 1997, 2001) $)^{10}$.

\section{Forma lógica}

Kolcaba (2003); afirmó que desarrolló la teoría del confort utilizando tres tipos de razonamientos lógicos: inducción, deducción y retroducción ${ }^{11}$.

\section{Inducción}

Consiste en elaborar generalizaciones a partir de un número de casos específicos observados (Bishop, 2002). Cuando las enfermeras están seguras de su práctica y la enfermería como disciplina cumple con sus objetivos entonces se conocen los conceptos implícitos y explícitos, términos, afirmaciones y supuestos que subyacen en su práctica. Cuando las enfermeras están estudiando, quizás que se les pide que hagan un diagrama de su práctica (como la doctora Rosemary Ellis le pidió a Kolcaba que hiciera), lo que es un ejercicio engañoso.

La situación a finales de la década de 1980 era la siguiente:

\section{Deducción}

Es una forma de razonamiento lógico que infiere conclusiones específicas a partir de premisas o principios más generales; va de lo general a lo específico (Bishop, 2002). El estadio deductivo del desarrollo de la teoría relacionó la comodidad con otros conceptos, y dio lugar a la teoría. Se utilizó el trabajo de tres teóricas: Orlando, Henderson, Paterson Zderat, para la definición de comodidad, por lo que Kolcaba tuvo que buscar otro espacio común para unificar alivio, tranquilidad y trascendencia. Se dió cuenta de que se necesitaba un marco conceptual más abstracto y general, que fuera coherente con la comodidad e incluyera un número manejable de ideas altamente abstractas. El trabajo del psicólogo Henry Murria (1938); cumplía los criterios para elaborar un marco en el que se situarán los conceptos enfermeros de Kolcaba. Su teoría trataba sobre las necesidades humanas, por tanto, podía

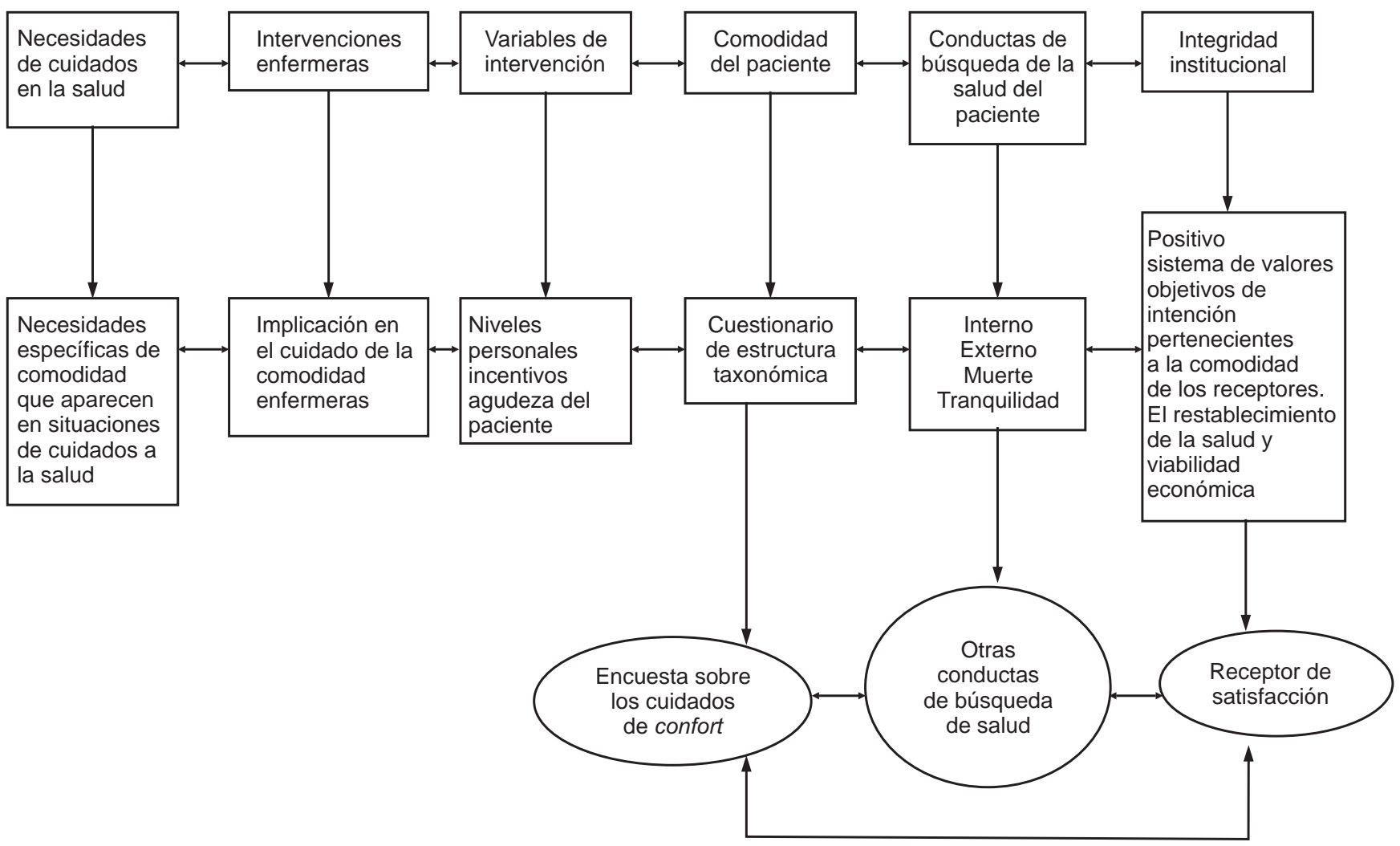

DE Kolcaba, K. Teoría del Confort adaptada a la investigación de resultados. Evolution of the midrange theory of comfort for outcomes research. Nursing Outlook 2001;49 (2), (86-92). 
aplicarse a los pacientes que experimentaban múltiples estímulos en situaciones estresantes de cuidado a la salud. Ése fue el estadio deductivo del desarrollo de la teoría, empezando por construcción y subestructuración teóricas generales y abstractas para descender a niveles más específicos que incluyen conceptos de la práctica enfermera.

\section{Retroducción}

Es una forma de razonamiento que origina ideas; se aplica a campos con pocas teorías disponibles (Bishop 2002), resulta útil para seleccionar fenómenos que pueden desarrollarse con mayor profundidad y probarse. Este es el caso de la investigación de resultados que, hasta la fecha, se centra en recoger datos para medir resultados seleccionados, y relacionar con los resultados de los tipos de protocolo enfermeros, médicos, institucionales o comunitarios. El uso de un marco teórico enfermero para la investigación de resultados potenciaría está área de la investigación enfermera, ya que la práctica basada en la teoría permite que; las enfermeras que diseñen intervenciones coherentes con los resultados deseados y aumenten la posibilidad de encontrar resultados significativos; para los objetivos deseados proporcionarían datos a las instituciones respectivas y a los creadores de estratégias sobre la importancia de la enfermería en el actual mercado competitivo.

\section{Patología}

Meningioma grado I de la hoz del cerebro, con efecto de masa, que comprime lóbulo frontal y parietal.

\section{Meningioma}

Los meningiomas son los tumores benignos más comunes del cerebro (95\% ); sin embargo, también pueden ser malignos. Surgen a raíz de la aracnoides de meninges y representan alrededor del $15 \%$ de todos los tumores cerebrales primarios; son más frecuentes en mujeres que en los varones (2:1), tienen una incidencia máxima en la sexta y séptima décadas. La mayoría de los casos son esporádicos, si bien algunos son familiares; se han registrado algunos indicios de que las personas que han sufrido radiación al cuero cabelludo están en situación de riesgo para el desarrollo de meningiomas. Las más frecuentes mutaciones genéticas que participan en los meningiomas son: mutaciones en la inactivación, neurofibromatosis, dos genes (esmerejón) en el cromosoma 22 $q^{12}$.

\section{Patógena}

Los meningiomas surgen de las células de la aracnoides, la mayoría de estos tumores están cerca de la vecindad de los senos venosos, este es el sitio de mayor prevalencia de la formación de meningiomas. Son más frecuentes adjuntos a la duramadre en la superficie parasagital de los lóbulos frontales y parientales, a lo largo de la cresta esfenoidal, en las ranuras olfatorias, la región silviana, en cerebelo superior a lo largo de la hoz cerebral, en el angulopontocerebeloso y la médula espinal. El tumor suele ser de color gris, muy bién circunscrito, asume la forma del espacio que ocupa. Son por lo general en forma de cúpula, también se presentan en la duramadre ${ }^{13}$. Histológicamente, las células son relativamente uniformes, con una tendencia a rodearse unos a otros, formando verticilos y psammoma órganos (concreciones de láminado cálcico). Tienen tendencia a calcificarse y son altamente vascularizados.

\section{Manifestaciones clínicas}

Pequeños tumores (por ejemplo $<2.0 \mathrm{~cm}$ ), suelen ser incidentales en la autopsia sin haber causado síntomas, los tumores más grandes causan síntomas en función del tamaño y ubicación las manifestaciones más importantes son: problemas en la coordinación, espasticidad progresiva, debilidad en las piernas, incontinencia, problemas de sensibilidad, afasia y aumento de la presión intracraneana (PIC).

\section{Diagnóstico $^{14}$ \\ - TAC, \\ - RM (con gadolinium) \\ - Arteriografía (por que son extra axial y vascularizados) \\ - Punción lumbar (proteínas elevadas)}

\section{Tratamiento}

- Cirugía: pueden ser resecado quirúrgicamente si el tumor es superficial en la duramadre y si este es de fácil acceso.

- Transarterial: por embolización

- Radioterapia: gamma knife o tratamiento de rayos de protones, en casos de tumores no resecables o inoperables.

- Quimioterapia: en caso de tumores malignos con antiprogestin.

\section{Marco metodológico}

Se trata de un estudio de caso, según las características de este proceso de acuerdo a lo que dice «...estudio de caso ${ }^{15}$, es el método de investigación que tiene por objeto comprender las conductas sociales de pequeños grupos o individuos concretos, a través de la observación en profundidad...», en este caso se trata al grupo determinado, una familia «X» de la colonia estrella del sur Iztapalapa D.F.

\section{Tipo de investigación}

Investigación descriptiva; comprende descripción, registro, análisis e interpretación de la naturaleza actual, y composición de los fenómenos. El enfoque se hace sobre conclusiones 
dominantes o sobre como una persona, grupo o cosa se conduce en el presente (Best, J. W., citado por Tamayo).

\section{Población y muestra}

Está constituida por una familia de la colonia estrella del sur Iztapalapa D.F., formada por padre, madre y dos hijos de $25 \mathrm{y}$ 24 años, de sexo masculino y femenino respectivamente.

Técnicas e instrumento de recolección de datos

Para la elaboración de este estudio, se seleccionó: el Cuestionario General de Confort desarrollado por la doctora Katharine Kolcaba, que consta de 28 ítem.

\section{Medidas de comodidad}

Para lograr esto, el familiar se le acerca al paciente, lo puede tocar, tomar de la mano, abrazar, besar, sonreírle, hablarle en forma suave y afectuosa. Escucharlo con atención, decirle palabras de aliento. Mirarlo con ternura. Realizar actividades de distracción como: leer, ver televisión y jugar. Así como brindarle información de acuerdo con sus necesidades (salud, religión, familiares, etcétera).

\section{Necesidad de comodidad física; medidas de comodidad}

Cambios de posición

- Lavarse las manos

- Ayudar a realizar cambios de posición, permitidos según el estado de salud de cada paciente

- Girar el cuerpo del paciente hacia el lado derecho, izquierdo o dejar boca arriba

- Asegurarse que no queden cables y otros dispositivos haciendo presión en la piel del paciente, (la enfermera se encargará de fijar y asegurar todos los dispositivos utilizados para monitorización del paciente, para que no se suelten con el movimiento).

- Tener las cosas personales del paciente en el cuarto y objetos que el quiera tener y que hagan el cuarto más hogareño.

- Adecuar la silla o cama según las necesidades del paciente.

\section{Hipótesis}

La intervención educativa hacia el familiar de la persona sometida a un proceso neuroquirúrgico influye de manera positiva para mejorar confort y calidad de vida.
Variables de estudio

\begin{tabular}{l|l}
\hline \multicolumn{1}{c|}{ Necesidad de comodidad } & Medidas de comodidad \\
\hline Comodidad social y psicoespiritual & - Estar cerca del paciente \\
- Seguridad & - Tomarle la mano \\
- Confianza & - Tener contacto físico: abrazar, besar \\
- Tranquilidad & - Sonreírle \\
- Afecto & - Hablarle en forma suave y afectuosa \\
- Apoyo & - Decirle palabras de aliento \\
- Compañía & - Escucharlo con atención \\
& - Mirarlo con ternura \\
& - Actividades de distracción: leer, ver televisión, \\
& - jugar, etc. \\
& - Proporcionar información según las necesidades \\
& específicas. \\
\hline Comodidad física & - Cambios de posición \\
& - Realizar masaje \\
& - Lubricar la piel \\
& - Tener ropa de cama limpia y sin arrugas \\
\hline Comodidad ambiental & Luz apropiada \\
- Proporcionar un ambiente agradable & - Temperatura adecuada \\
& - Eliminar el ruido \\
\hline & - Tener las cosas personales del paciente \\
& - Adecuar la silla o cama, más blanda o dura. \\
\hline
\end{tabular}

Procedimiento

En el presente estudio se midió la intervención de enfermería para la orientación con la guía de confort e influye en grado de comodidad de la persona en este caso de G.H.M. hospitalizado en el servicio de Neurocirugía del Instituto Nacional de Neurología y Neurocirugía (INNN), durante el 31 de octubre del 2007 al 2 de noviembre de 2007. Se aplicó el instrumento de valoración al bajar de recuperación, al día siguiente se le proporciona la guía a los familiares, al tercer día se vuelve aplicar el mismo instrumento de valoración al paciente. Se utiliza el Proceso Enfermero: se valora, diagnóstica, planifica intervenciones, ejecutan y evalúan.

\section{Historia clínica de enfermería}

Datos de identificación

Nombre: G.H.M.

Servicio: Unidad de Neurocirugía.

No. de cama: 146

Fecha de ingreso: 8 de octubre de 2007.

Edad: 25 años

Sexo: masculino

Estado civil: soltero.

Escolaridad: ingeniero en computación

Ocupación: trabaja en la empresa Liverpool, en la Unidad de

Sistemas

Religión: católica.

Nacionalidad: mexicano

Lugar de procedencia: D.F.

Domicilio: Calle Flores Magno \# 37. Colonia Estrella del

Sur. 09820 México, D.F.

Perfil del paciente

Ambiente físico. 


\section{Habitación}

a. Características físicas (iluminación, ventilación, etc.), luz natural y artificial adecuada a las necesidades del hogar.

b. Casa propia y familiar

c. Construcción: concreto

d. Número de habitaciones: tres recamaras, sala-comedor y cocina.

e. Animales domésticos: un perro

Servicio sanitario

a. Agua: intradomiciliaria, suficiente, día y noche.

b. Control de basura: recolección diaria

c. Eliminación de desechos: drenaje

d. Iluminación: todas las habitaciones y alumbrado público.

e. Pavimentación: todas las calles

Vías de comunicación

a. Teléfono particular

b. Medios de transporte: autobús, metro, camión, taxi

c. Recursos para la salud: médico particular, S.S.

Hábitos higiénicos: aseo: baño diario, lavado de manos las veces necesarias y lavado bucal tres veces al día o las necesarias, cambio de ropa diario.

Alimentación desayuno: leche, cereal, una pieza de pan, fruta

Comida: sopa, carne (tres veces por semana), agua, frijoles y fruta

Cena: pan, café o leche

\section{Eliminación:}

Vesical: hasta tres veces al día de color amarillo cristalino, de olor característico.

Intestinal: una vez al día de color y olor característico.

Descanso: por las tardes y noche sueño tranquilo

Composición familiar

\begin{tabular}{l|l|l|l}
\hline Parentesco & \multicolumn{1}{|c|}{ Edad } & \multicolumn{1}{|c}{ Ocupación } & Participación económica \\
\hline Padre & 52 & Obrero & \$6.000 mensuales \\
Madre & 48 & Hogar & Ninguna \\
Hijo & 25 & Ingeniero en sistemas & $\$ 15.000$ mensuales \\
Hija & 24 & Secretaria & $\$ 8.000$ mensuales \\
\hline
\end{tabular}

Dinámica familiar: hay comunicación adecuada con sus padres así como con su hermana.

Dinámica social: tiene amigos (as), así como convivencia con vecinos y compañeros de trabajo.

Comportamientos: se considera una persona sociable, animada, con metas y proyectos.
Rutina cotidiana: levantarse temprano, 6:00 a.m. hacer ejercicio, ducharse, desayunar e ir a trabajar 9: 00 a 17:00 hs ver televisión, ir al cine o salir de vez en cuando con los amigos.

Problema actual: meningioma (neoplásia de hoz cerebral grado I). El paciente narra que inicio hace tres meses con dolores de cabeza, estos fueron incrementandose más a finales de septiembre, el dolor no cedía con analgésicos asiste a médico particular quien decide referirlo al INNN, en donde le realizan exploración neurológica, TAC.

Antecedentes familiares patológicos: diabetes mellitus padre: cardiopatía; madre: con obesidad, su abuela materna muere de cáncer.

Antecedentes personales patológicos: cuadros frecuentes de faringoamigdalitis de niño, inicia hace tres meses con dolores de cabeza y es diagnósticado neoplásia, la cual requiere tratamiento quirúrgico, que es el motivo de su estancia hospitalaria.

Comprensión y/o comentario acerca de su padecimiento: está muy optimista y con mucha i.e. a que todo va a salir bien, ya que el médico le ha explicado, que puede reincorporarse a sus deberes en un meses después de la cirugía.

Participación del paciente y la familia en el diagnóstico, tratamiento y rehabilitación: lo visitan diariamente y están al pendiente de sus necesidades, comentan su padecimiento con el médico, tratando siempre de mantenerse informados de la evolución del paciente, se le brinda apoyo, seguridad y tranquilidad a G.H.M.

\section{Exploración física}

Habitus exterior: persona del sexo masculino de edad aproximada a la cronológica (25 años), con actitud somática adaptada, con nebulizador, actitud psicológica: inquieto e intranquilo, por molestia oro traqueal, con ligero déficit sensitivo de hemicuerpo izquierdo, coeficiente intelectual de 8.5, de nivel socioeconómico medio, originario del D.F., la marcha no se puede apreciar porque está en las primeras $24 \mathrm{hs}$ de posoperado.

Aspecto emocional: se muestra inquieto al tener dolor en lado de la herida quirúrgica y manifiesta que después de la cirugía no ha visto a sus familiares, tiene molestias en garganta para deglutir, así como de lado izquierdo de su cuerpo no hay mucha fuerza. Aquí se le ha explicado que las visitas son después de las 15:00 hs el baja de recuperación a las 10:00 hs, que duerma y este tranquilo ya que no deben tardar sus familiares.

Somatometría: peso: $70 \mathrm{~kg}$, talla $1.75 \mathrm{~cm}$, pulso $88 \mathrm{~min}$, ritmo regular, respiraciones $22 \mathrm{~min}$, temperatura $36.5^{\circ} \mathrm{C}$, tensión arterial 120/80 mm/hg. 
Piel: tegumentos con ligera pálidez, pero de buen aspecto, con sensibilidad al dolor.

Palpación: no se realiza palpación de cabeza por el procedimiento quirúrgico, a la observación cráneo normocéfalo con herida quirúrgica de lado derecho, con vendaje tipo capelina y baja de recuperación con drenaje bilateral 1: subgaleal 2: subdural, con colección de líquido hemático más en el drenaje de tipo subgaleal aproximadamente de $50 \mathrm{ml}$. En el de tipo subdural de $30 \mathrm{ml}$, con ligera rígidez de cuello.

Ojos: sin presencia de inflamación; hidratación y coloración aceptables, con equilibrio muscular, con pupilas isocóricas normoreflexicas, con muy buena agudeza visual.

Nariz: sin desviación del tabique nasal, sin datos de secreción.

Boca: mucosas hidratadas, se aprecian todas sus piezas dentales, sin datos de gingivitis y estomatitis, sólo refiere ligero dolor al deglutir sus líquidos claros. Aquí se le explica que estuvo orointubado durante algunas horas para el procedimiento quirúrgico, que quizás está es la causa, y que va a disminuir la molestia.

Garganta: se le pide que abra la boca para observar la garganta y ver cuál es la causa de la molestia; se aprecian las dos amígdalas sin datos de inflamación, faringe con buena coloración y úvula central.

Oídos: izquierdo y derecho de características normales, con buena audición.

Cuello: con ligera molestia al movimiento, aquí se le explicó que es por el tiempo que estuvo su cabeza en determinada posición por la cirugía, no se palpan ganglios submaxilares, submentonianos, cervicales, retroaurculares. Con pulsaciones carótidas adyacentes a la traquea que cruzan la línea media del músculo esternocleidomastoideo, se puede sentir, pulsar, pero no se les puede ver, las venas yugulares internas y externas son laterales al músculo esteronocleidomastoideo; inmediatamente por arriba de la clavícula, se las puede ver pero no se pueden sentir y pulsar, con buena localización traqueal.

Tórax: con simetría de movimientos respiratorios, a la auscultación se pueden apreciar sonidos de tonalidad regular y de duración igual regular por el paso de aire a través de los alvéolos, con 22 respiraciones por minuto, sin datos de estertores.

Corazón: con aceptable ruidos cardiacos, se escucha muy bien, con buen ritmo y regularidad en cuanto a la sístole y diástole de 88 por min.

Abdomén: no se palpan presencia de masas anormales, con adecuados ruidos peristálticos que se producen por el paso del aire y líquido a traves del estómago e intestinos, percudiéndose y palpándose vejiga llena.

Extremidades miembros superiores: simétricos, con catéter largo y central miembro superior izquierdo, conectado a solución de base para 18 hs, a 60 cc por hora con bomba de infusión; (ya que ha tolerado líquidos v.o.), con parche en arteria radial ya que tenía línea arterial para toma de muestras de laboratorio durante y después de la cirugía para controles de laboratorio. Y a piso bajan sin líneas arteriales, sólo venosas, con falanges digitales: meñique cordial, anular, índice y pulgar con muy buen llenado capilar, se palpa adecuado pulso radial, cubital y braquial aceptable crecimiento de las uñas de ambas manos.

Miembros inferiores: simétricos, sólo refiere tener levemente disminuida la fuerza en miembro inferior izquierdo.

\section{Datos complementarios}

Exámenes de laboratorio

Fecha: 25 de octubre de 2007

Hematología: paciente normal

Hemoglobina $\quad 14 \quad 14-15$

Hematocrito $\quad 40 \quad 38-40$

C.M.H.C. $\quad 36.8 \quad 36-38$

Leucocitos $\quad 5.000 \quad 4000-6000$

Nota: las cifras de los componentes sanguíneos están en limites normales, muy bien controlados durante el procedimiento quirúrgico.

Química sanguínea

\begin{tabular}{lcc}
\hline Componente & cifra \% & normal \% \\
\hline Química & $114 \mathrm{mg}$ & $76-115 \mathrm{mg}$ \\
Urea & $28 \mathrm{mg}$ & $20-40 \mathrm{mg}$ \\
Creatinina & $0.8 \mathrm{mg}$ & $0.8-1.5 \mathrm{mg}$ \\
Bilirrubinas totales & $0.6 \mathrm{mg}$ & $0.6-1.7 \mathrm{mg}$ \\
Bilirrubinas indirecta & $0.4 \mathrm{mg}$ & $0.1-1.0 \mathrm{mg}$ \\
Colesterol & $165 \mathrm{mg}$ & $131-300 \mathrm{mg}$ \\
Proteínas totales & $6.3 \mathrm{~g}$ & $6.4-8.4 \mathrm{~g}$ \\
Albuminas & $4.0 \mathrm{~g}$ & $3.8-5.0 \mathrm{~g}$ \\
Globulinas & $2.3 \mathrm{~g}$ & $2.5-3.0 \mathrm{~g}$
\end{tabular}

Exámenes de gabinete

- Rayos $X$ de tórax: la traqueotomía es central, la silueta cardiaca de tamaño y contorno normales. Los senos cortos y cardiofrénicos se muestran libres y las estructuras óseas visibles de tejidos blandos, no muestra alteraciones.

- Tomografía axial computalizada: tomografía simple y contrastada de la base de la convexidad, identificándose las estructuras óseas y cavidades neumáticas de aspecto normal, se aprecian ventrículos de tamaño normal, así como zona de calcificación intracraneana con la administración 
de medio de contraste se refuerza en forma intensa en los repliegues aracnoideos tanto de la parte media, en la hoz del cerebro y desplazando en la unión fronto-temporal.

\section{CONCLUSIÓN PARTICULAR}

Probable meningioma de la hoz, de origen a determinar cráneo, hipertensivo.

Proceso de atención de enfermería. Proporciona la base para el control operativo y el medio para sistematizar y hacer investigación en enfermería. Asegura la atención individualizada.

\section{OBJETIVO}

Proporcionar un sistema que pueda cubrir las necesidades individuales del paciente, familia y comunidad.

Ventajas del proceso de atención de enfermería: a la persona le permite participar en su propio cuidado; garantizando la respuesta a sus problemas reales y potenciales, ofreciendo atención individualizada continua y de calidad, de acuerdo con la priorización de sus problemas. El uso del proceso de atención de enfermería da como resultado un plan que describe las necesidades de atención y cuidados de enfermería para cada sujeto de atención.

Indicadores del proceso de enfermería: el propósito del proceso es dar un marco de referencia dentro del cual las necesidades del sujeto de atención, familia y comunidad puedan ser atendidas en forma integral ${ }^{19}$. Se organiza en cinco fases secuenciales, aunque en la práctica pueden darse simultáneamente. El proceso de atención que tiene como propiedades ser más dinámico, flexible, continuo, individualizado, participativo y con un fin determinado. Además, se debe basar en un marco teórico.

\section{Fases del proceso de atención de enfermería}

Como se mencionó con antelación, el proceso se organiza en cinco fases cíclicas interrelacionadas y progresivas: 1. Valoración. 2. Diagnóstico de enfermería. 3. Planeación. 4. Ejecución-intervención, y 5. Evaluación ${ }^{20}$.

\begin{tabular}{|c|c|c|c|c|c|}
\hline Problema & $\begin{array}{l}\text { Diagnóstico de } \\
\text { enfermería }\end{array}$ & Meta & $\begin{array}{l}\text { Órdenes } \\
\text { de enfermería }\end{array}$ & Evaluación & Revaloración \\
\hline $\begin{array}{l}\text { Incapacidad para eliminar } \\
\text { secreciones }\end{array}$ & $\begin{array}{l}\text { Limpieza ineficaz de las vías } \\
\text { aéreas } r / c \text { abundancia y } \\
\text { viscosidad de las secreciones }\end{array}$ & $\begin{array}{l}\text { Mejorará la } \\
\text { permeabilidad de la } \\
\text { vía aéreas en } 72 \mathrm{~h}\end{array}$ & $\begin{array}{l}\text { Aumentar la ingesta de líquidos a } 2 \text { o } 3 \mathrm{~L} \text { al día, si no hay } \\
\text { contraindicación } \\
\text { Enseñar el método correcto para la tos eficaz }\end{array}$ & $\begin{array}{l}\text { Utiliza el método correcto } \\
\text { para realizar la tos eficaz } \\
\text { La tos es productiva }\end{array}$ & $\begin{array}{l}\text { Si las intervenciones de enfermería } \\
\text { no resuelven totalmente el problema, } \\
\text { agregar la realización de drenaje } \\
\text { postural, percusión y vibración } \\
\text { Revisión médica }\end{array}$ \\
\hline $\begin{array}{l}\text { Incapacidad para } \\
\text { realizar las actividades de la } \\
\text { vida diaria (AVD) }\end{array}$ & $\begin{array}{l}\text { Intolerancia de la actividad r/c } \\
\text { inadecuada oxigenación y fatiga }\end{array}$ & $\begin{array}{l}\text { En una semana será } \\
\text { capaz de realizar las } \\
\text { AVD sin manifestar } \\
\text { fatiga }\end{array}$ & $\begin{array}{l}\text { Evitar actividades y factores que aumentan la demanda de } \\
\text { oxígeno (temperatura extrema, estrés y otros) } \\
\text { Recomendar técnicas de ahorro de energía } \\
\text { Acompañarlo en las actividades que demandan esfuerzo } \\
\text { Aumentar gradualmente las actividades según tolerancia } \\
\text { Enseñar técnicas de respiración eficaz } \\
\text { Después de la actividad buscar respuestas anormales al } \\
\text { aumento de la actividad (taquipnea, taquicardia, hipotensión o } \\
\text { vértigo) }\end{array}$ & \begin{tabular}{|l|} 
Realiza las AVD. Sin fatiga \\
No presenta respuestas \\
anormales con las AVD \\
Coordina las actividades con \\
la respiración eficaz
\end{tabular} & \\
\hline Ansiedad & $\begin{array}{l}\text { Ansiedad r/c dificultad para } \\
\text { respirar }\end{array}$ & $\begin{array}{l}\text { En dos días } \\
\text { expresará sentirse } \\
\text { más tranquilo }\end{array}$ & $\begin{array}{l}\text { No dejarlo solo durante los períodos de dificultad respiratoria } \\
\text { Animarlo a usar las técnicas respiratorias durante los } \\
\text { momentos de ansiedad aguda } \\
\text { Enseñarle técnicas de relajación cortas y sencillas } \\
\text { Proporcionar un ambiente tranquilo y seguro }\end{array}$ & \begin{tabular}{|l|} 
Expresa aumento en su nivel \\
de bienestar psicológico y \\
fisiológico \\
Se observa \\
Tranquilo
\end{tabular} & $\begin{array}{l}\text { Si no se logra la meta: } \\
\text { reducir estímulos ambientales para } \\
\text { favorecer la relajación } \\
\text { Ofrecer ayuda si la necesita } \\
\text { Enseñarle a concentrarse en la } \\
\text { respiración diafragmática con labio } \\
\text { fruncido }\end{array}$ \\
\hline
\end{tabular}

\section{CONCLUSIONES}

Además, de los diagnósticos de enfermería desarrollados en el ejemplo, deben explorarse otros problemas comunes en las personas con esta enfermedad, de los cuales pueden surgir otros diagnósticos de enfermería como pueden ser: 1. Riesgo de alteración de la nutrición (por defecto r/c anorexia secundaria a disnea, halitosis y fatiga), 2. Alteración de la comunicación verbal r/c disnea, 3. Trastornos del patrón del sueño r/c tos e incapacidad para adoptar la posición de decúbito y 4. Riesgo de alteraciones del mantenimiento de la salud r/c conocimiento insuficiente del problema, tratamiento farmacológico, prevención de la infección, tratamiento nutri- cional, descanso y actividad, ejercicios respiratorios y cuidados domésticos. Después de identificar y priorizar los problemas, se debe recurrir a una lista actualizada de diagnósticos de enfermería aprobados por la NANDA, para proceder a elaborar los diagnósticos pertinentes.

\section{BIBLIOGRAFÍA CONSULTADA}

1. Marriner-Tomey Ann, Raile Alligow Martha. Modelos y teorías en enfermería. 6a. Ed. Harcourt Brace. España 2007.

2. Grooper L. Promoting health by promoting comfort. Nursing Forum 1992;27(2):5-8.

3. Kolcaba K. An analysis of the concept of comfort. Advanced Nursing 1991a;16:1301-10. 
4. Kolcaba K. The art of comfort care. Image: Nursing Scholarship 1995;27(4):287-9.

5. Kolcaba K. A theory of holistic comfort for nursing. Journal of Advanced Nursing 1994; 6(19):1178-84.

6. Kolcaba K, Fischer E. A holistic perspective on comfort care as an advance directive. Critical Care Nursing Quaterly 1996;18(4):66-76.

7. Roger P, Michael J. Neurología Clínica. Ed. El Manual Moderno. México, D.F.

8. Decq P. Keravel, Velasco F. Neurocirugía. $1^{\text {a }}$ Edición Ed. Marketing/ Elipses. París Francia. 2000.
9. Hernández Sampieri Roberto. Metodología de la Investigación $4^{\mathrm{a}}$ Ed. Editorial Mc Graw - Hill. Interamericana. México 2006.

10. Iyer P, Tapich B, Bernocchi-Losey D. Proceso de enfermería y diagnóstico de enfermería. México: Interamericana McGraw-Hill; 1993:13.

11. López P. Proceso de enfermería. Rev Iberoamericana de Geriatría y Gerontología 1994;10:37-8.

12. Atkinson L, Murray ME. Proceso de atención de enfermería. $2^{\mathrm{a}}$ ed. México. El Manual Moderno; 1983.

13. Luis Ma.Teresa. Diagnósticos de enfermería. Aplicación práctica. Revisión Crítica y guía práctica. $7^{\text {a }}$ Edición Editorial Masson.1991. 